tion in the normal way is obtained for a certain range of dose-rates, but for less than a certain pre-set limit cancellation is provided. Such a system with an alarm operating on the integrated dose appears to offer many advantages over the conventional systems. In a complete system three identical detector excursion detector units are used operating independently. The output of these units is connected into a two-out-of-three matrix such that at least two units must alarm to give a true excursion signal and initiate personnel evacuation.

D. E. Barnes (Atomic Weapons Research Establishment, Aldermaston) spoke about the important problem of monitoring for airborne plutonium and considered the instrumental systems under three headings: $(a)$ direct, in which the plutonium and background particles are collected and measured together; $(b)$ selective, in which an attempt is made to collect only the plutonium dust; $(c)$ compensative, in which the background and plutonium dusts are collected together, but the counting system is designed to remove the 'counts' due to background before readout. D. S. Hiorns and G. G. Dale (both of the Central Electricity Generating Board, London) each contributed a specialist paper on "Health Physies Instruments", the latter making it clear that while the majority of instrumentation problems in this field had been satisfactorily solved, two problems still remained for which an improved solution was still wanted. These were: $(a)$ a dose-rate meter capable of monitoring gamma-radiation down to a few times background; $(b)$ a fast neutron monitor capable of measurement in the presence of a large $\gamma$-radiation background.
Denis TAYLOR

\title{
TRENDS IN DENTAL ANTHROPOLOGY
}

A

NTHROPOLOGICAL interest in teeth until a fow years ago lay primarily in using details of dental form to trace phylogenetic relationships among Primates present and past, secondly in describing the frequencies of dental characters in various human populations. How far and in what directions dental anthropology has progressed was well shown by the 1961 symposium of the Society for the Study of Human Biology, held at the British Museum (Natural History) on November 17. In a word, the present approach is much more biological than in the older descriptive studies; for that reason some of the papers will be of interest also to those concerned with human genetics, embryology and growth.

Dr. P. M. Butler directed attention to the difficulties of inferring phylogenetic relationships from dentition. In fossil and living Primates too little is known of variation within a population; of the function of, say, details of cusp pattern, and hence of the adaptive significance of observed variations; of individual development, and particularly the extent of ontogenetic variation; of the genetic basis of dental morphology and development. It was still not possible to say which hypothetical evolutionary progressions could have oceurred and which not. Some of his own work on the fiold theory of dental development provided some relevant indications. The shape of the crown of a mammalian tooth and all its details derive ontogenetically from the folding of a sheet of epithelium, which forms the internal surface of an ectodermal enamel organ. This can first be distinguished as a bud on the dental lamina. Teeth buds appear in a characteristic order for a given species, shown by plotting their appearance against embryo size. The sequence is not from mesial to distal but is determined by pre-existing 'tooth districts'; one such controls the anterior teeth, and within it the bud of the first incisor precedes that of the second, etc.; another the posteriors, so that the graph for the whole primary series shows something approaching a wave form. Comparison of the graphs for some primates with that of an insectivore shows that the latest-appearing buds of the latter have been lost. The graphs show, moreover, that teeth developing at the same time can be morphologically different, while teeth developing at different times may be similar if they are next to each other in the jaw. It seems that in the embryo jaw there is a continuous morphogenetic ficld or gradient controlling the development of the teeth germs according to their position within it; this field exists before any teeth buds commence to form, and may be undor the influence of one set of genes; another set of genes may be postulated which influence the way in which tooth germs respond at any given point in the field.

Development was also the theme of the paper by Dr. B. S. Kraus (Seattle), who spoke on the morphogenesis of the deciduous molars in man. From an examination of a large number of foetuses it appears that for each molar there is a characteristic pattern of calcification of the crown. In the lower first molar the sequence of appearance of the calcification centres is protoconid, metaconid, hypoconid, entaconid, hypoconulid. Only when all five centres have appeared does bridging between them commence; again this occurs in a regular sequence, linking first the protoconid and hypoconid, then in succession the hypoconid and hypoconulid, the protoconid and metaconid, the hypoconulid and entaconid. In the lower second molar the order of appearance of the centres is similar but the bridging sequence is different. The upper first molar is very different and developmentally is reminiscent of a bicuspid. Not only does each molar have its own characteristic pattern of calcification, but these also are interrelated so that a definite pattern is discornible when all four molars in a single fœtus are considered together. The series of fotuses was sufficiently large to demonstrate that there were relatively few other sequences which any variants would follow. Photomicrographs of the undulations of the soft tissue surface of the crown showed how calcification is affected by them. Di. Kraus concluded by directing attention to the probability that these sequences of calcification were under genetic control, and the possibility of using incidence of variants in radiation effect studies.

Occlusion and malocclusion in the Primates was discussed by Dr. J. R. E. Mills (London). Study of dynamic occlusion (that is, as in normal chewing movements) in the gorilla showed that the large canines do not prevent lateral mandibular movement as formerly thought, but merely limit it. Balanced occlusion, in which on one side of the jaw the lower buccal cusps of the posterior teeth come into relation with the upper lingual cusps, then slide across the crown surface until the lower lingual oppose the 
upper buccal, is produced by rotation about the two mandibular condyles alternately. Rotation about the condyles occurs, to varying degrees, throughout the Primates down to Tupaia notwithstanding differences in teeth shape. In man, where there has been reduction in size of jaw, reduction of prognathism and change in jaw shape, jaw movement and the relations between cusps remain basically the same as in apes. Malocclusion is found throughout the Primates, and most frequently in those where the cusps of the posterior teeth are low and where it interferes least with function; incidence is higher and the degree more severe in animals raised in captivity. Man has the highest incidence of malocclusion, though there is marked variation between races, and indeed sometimes between small local populations. While severe malocclusion is also observed in many Primate species, it is particularly frequent in civilized man. This may be duo to environmental influences on bone growth; to intermixture of populations, giving rise to incongruities of parts of the skull or size disorepancies between teeth and jaw; or to the greater likelihood of survival of individuals with severe malocelusion.

Dr. A. A. Dahlberg (Chicago) pointed out that the present minimizing of the importance of teeth in Western peoples, the reliance on foods prepared to require little mastication and on artificial substitutes for teeth, tended to lead to underestimation of their importance under primitive conditions. The dentition of American Indian peoples, whose morphological and metrical features were reviewed, shows considerable heterogeneity from group to group. He suggested that the observed differences could provide olues to the adaptive significance of variations in dental detail. Primitive diets containing considerable abrasive material led to early obliteration of crown patterns, which, it could be argued, were therefore of littlo selective significance; yet the experimental study of tooth wear showed that the tooth volume expenditure per calorie derived from a given food varies considerably with the texture and other properties of the food and with morphological details of the dentition. In primitive man subject to marginal availability of food, containing, moreover, appreciable indigestible matter, slight dental variations could well have affected survival.

Mr. D. Goose (Liverpool) reviewed some recent applications of metrical techniques. He illustrated the point, that in some dental studies the only approach available lay in measurement, by reference to problems of identification of fossil specimens; of differences in tooth size and shape between races; and of changes in jaw size, contrasting with the absence of change in tooth size, in the inhabitants of Britain since Romano-British times. Miss V. M. Carbonnell (London) reported variations in incidence and distribution of the shovel shape of incisors in Old World populations, pointing out that the decline in frequency in civilized man was compatible with the view that the function of this feature was to strengthen the incisor. Dr. V. Alexander directed attention to the elevated frequencies of double-rooted lower canines in European populations by comparison with nonEuropean. This phenomenon he interpreted as resulting from a forward extension of the morpho. genetic field responsible for the formation of more than one root in the cheok teeth.

Age assessment by dentition was discussed by Dr. A. E. W. Miles (London). Several newly developed methods allow the age of an infant to be estimated with a high degree of accuracy. In young infants when the neonatal growth line can be discerned, counting from it the number of cross-striations of the enamel prisms, or measuring the thickness of the deciduous dentine, gives estimates correct within weeks. Error increases with the age of the individual, and other dental details, for example, length of translucency of the root apex, or combinations of these. give better results. At later ages crown wear, which progresses at a fairly constant rate for each tooth, allows the functional age of a tooth to be assessed. Of about 150 Saxon skulls, in 26 the age could be estimated from the state of the immature dentition, and these provide a basis for assessing the rate of crown attrition. The third molars wear slightly more slowly than the second, and these again more slowly than the first permanent molars. By comparison of the amount of wear on a number of teeth, age of adults up to about 45-50 years old can be assessed. The method applied to the whole Saxon series shows the average age at death in this population to have been about 31 years.

With Prof. C. F. Ballard and P. O. Pedersen in the chair respectively in the morning and afternoon sessions the meeting proceeded smoothly. Most members of the audience found much that was new in most of the papers. There were, of course, topics of current interest which did not appear in the programme; for example, variations in dental growth among different human populations, secular and regional variations in dental disease incidence, the relation between chewing habits and dental structure. Yet there is a limit to what can be covered in a single day's discussion, and the organizer, Dr. D. R. Brothwell, is to be congratulated on a stimulating programme as representative of present trends in dental anthropology as time would allow.

D. F. ROBERTS

\section{THE VICKERS GROUP RESEARCH ESTABLISHMENT}

$\mathrm{L}$ ORD HAILSHAM, Minister for Science, visited 1 the Vickers Group Research Establishment in Sunninghill, near Ascot, on December 4, when he met the Boards of Directors of Vickers, Ltd., and Vickers Research, Ltd., and members of the Research Establishment. During his visit he was shown examples of the present lines of work, new research tools and some novel products which are results of the research work. This Establishment is the centre of the research organization of the Vickers Group of companies and is operated by the Research Compeny. It carries out both fundamental and applied research, the results of which are used by the companies of the Group when they are likely to yield profitable new products or processes. The present work inoludes. among others, medical, chemical, hydraulio and electron physics investigations.

The medical work is based on a combination of the disciplines of the biological and physical sciences. It is concentrated on the respiratory and circu- 